


\section{La ciudad como reflexión formal}

The city as a formal thinking

Juan Diego Camacho

ISSN (imp): 1390-4825

ISSN (e): 2477-9199
Fecha de recepción: 13/04/18 Fecha de aceptación: 15/05/18

\section{Resumen:}

El presente artículo reflexiona acerca de la obra de instalación titulada "Lo Que Será Será y Así Fue", exhibida en la muestra Cuerpos de Lengua - KIIL en el Museo de Arte Contemporáneo del Zulia (MACZUL) en Venezuela, en noviembre del 2017. A través de una ambientación arquitectónica, la obra visibiliza una realidad urbana que tienen en común las grandes ciudades de los países con dependencia económica y tecnológica de las potencias, que, a pesar de sus múltiples diferencias lingüísticas o culturales, comparten una tipología arquitectónica. Esta pieza remite a distintos conceptos de la ciudad, quizás abstractos e invisibles, como sus derechos, el territorio, el poder y la identidad, a la vez que señala al aparato estético del museo y la estética de lo bello.

\section{Palabras clave:}

Ciudad, territorio, poder, movilidad, hiperdegradado, arquitectura, instalación, ambientación

\section{Abstract:}

This article talks about the piece "Lo Que Será Será y Así Fue", that was part of the exhibition Cuerpos de Lengua -KILL in the Museum of Contemporary Art in Zulia, Venezuela, in November 2017. Through an architectonic installation, the piece shows a common urban reality in big cities from developing countries that, despite their cultural and linguistic differences, share an architectonic typology. This paper talks about several city concepts, perhaps abstract and invisible like their rights, their territory, the power, and the identity, while pointing out the museum aesthetic and the aesthetic of beauty.

\section{Key Words:}

City, territory, power, mobility, hiperdegradation, architecture, installation, ambientation.

\section{Biografía del autor:}

Juan Diego Camacho. Maracaibo, Venezuela, 1994. Estudió cuatro años Artes Plásticas y es licenciado en Diseńo Gráfico. Se ha desempeñado como preparador docente en la Facultad de Artes de la Universidad del Zulia. Actualmente es colaborador y facilitador en el MedialabUIO, en torno a temas de arte digital. Dentro de su proceso de producción e investigación le interesan los nuevos medios, el desarrollo front-end, la programación, la electrónica, la tecnología y la imagen técnica. 
El entorno urbano y la ciudad representan para el hombre la tentativa más satisfactoria de recrear el mundo en el que vive de acuerdo con su propio deseo. Pero si la ciudad es el mundo que el hombre ha creado, también constituye el mundo donde está condenado a vivir. Así, indirectamente y sin tener plena conciencia de la naturaleza de su obra, al crear la ciudad, el hombre se ha rehecho a sí mismo.

(Park, 1911, p.73)

\section{CONSIDERACIONES SOBRE LA OBRA}

"Lo Que Será Será Y Así Fue"es una obra enmarcada dentro del proyecto de intercambio KIIL" en conjunto con estudiantes de la Hochschule für Küste im Sozialen, de Ottersberg, Alemania, y la Universidad del Zulia, de Maracaibo, Venezuela, y realizada en noviembre del año 2017 . Este proyecto responde a las necesidades y problemas actuales del uso de las intervenciones artísticas en los diferentes campos de aplicación clínica, socio-pedagógicos y sociales. Como resultado, se realizó la exhibición Cuerpos de Lengua - KIIL, que hace referencia a dos cualidades dentro de la producción de las obras puestas en sala: por un lado, la importancia del cuerpo como forma y contenido de las obras, y, por otro, los límites y dificultades de la comunicación a través de los diferentes lenguajes de los integrantes, (alemán, español e inglés).

En este ensayo se desarrolla una reflexión acerca del marco teórico que engloba una de las obras dentro de la muestra. "Lo Que Será Será Y Así Fue" - pieza realizada por Ariane Holz, Jesús Madueño, Angel D. Leiva Valera y Juan Diego Camacho- es una construcción de diversos materiales y de objetos encontrados, en su mayoría, diferentes tipos de maderas, plásticos y telas, yuxtapuestos unos sobre otros creando una especie de edificación desproporcionada que permitía el tránsito de los espectadores a través de diferentes ambientes (VerFigura 1).

Uno de estos, (Ver figura 2 y figura 3) a través de la presencia y agrupación de algunas formas dentro del mismo espacio, remite a un tendedero: nos en-

1 KIIL (Kunstlerische Interventionen Im Lebenswelten) - Intervenciones Artísticas en los Mundos de Vida, título del proyecto de la HKS - Ottersberg.

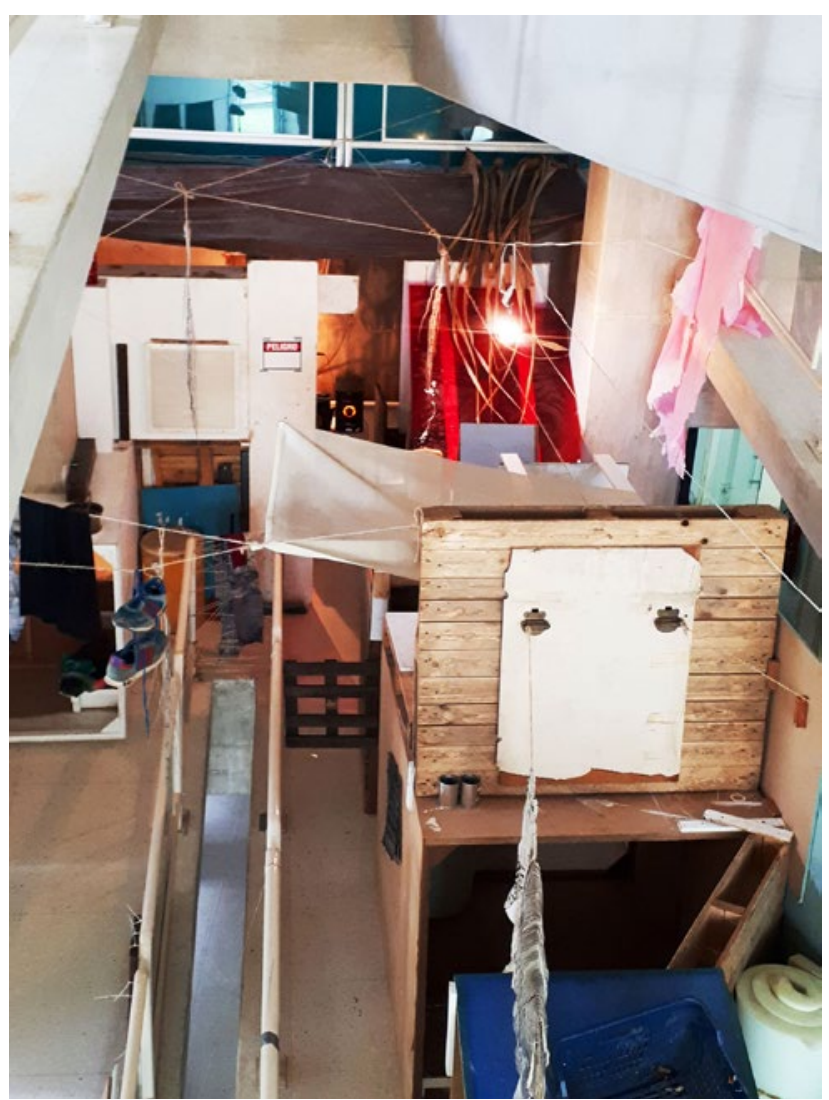

1. Imagen en picado de la pieza "Lo Que Será Será Y Así Fue" en la cual se nota la yuxtaposición de diferentes materiales que crean ambientes internos y externos por los cuales transitar o tenderse. Fotografía de Ariane Holz (2017).

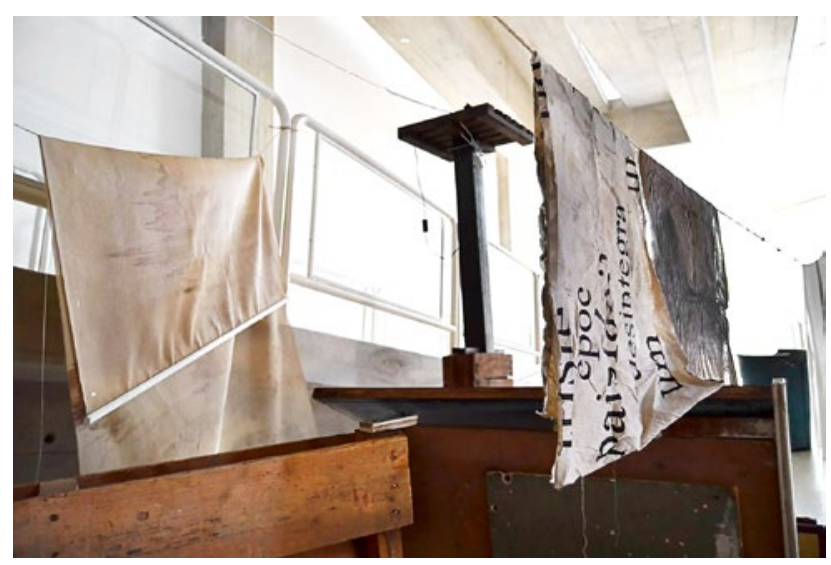

2. Registro de la obra "Lo Que Será Será Y Así Fue" donde se hallan algunas telas guindadas y entre ellas una construcción q

ve representaba un poste de electricidad de donde salían cables que estaban conectados a los bombillos de las áreas interiores de la pieza, fotografía de Ángel D. Leiva Valera (2017)

contramos con una cuerda que atraviesa el espacio en donde están colgadas algunas telas y demás elementos. El conjunto de estos espacios y de sus ele- 
mentos logra representar la estructura de una casa: un sitio donde sentarse, fumar, escuchar música a todo volumen o colgar la ropa. En resumen, un lugar donde habitar.

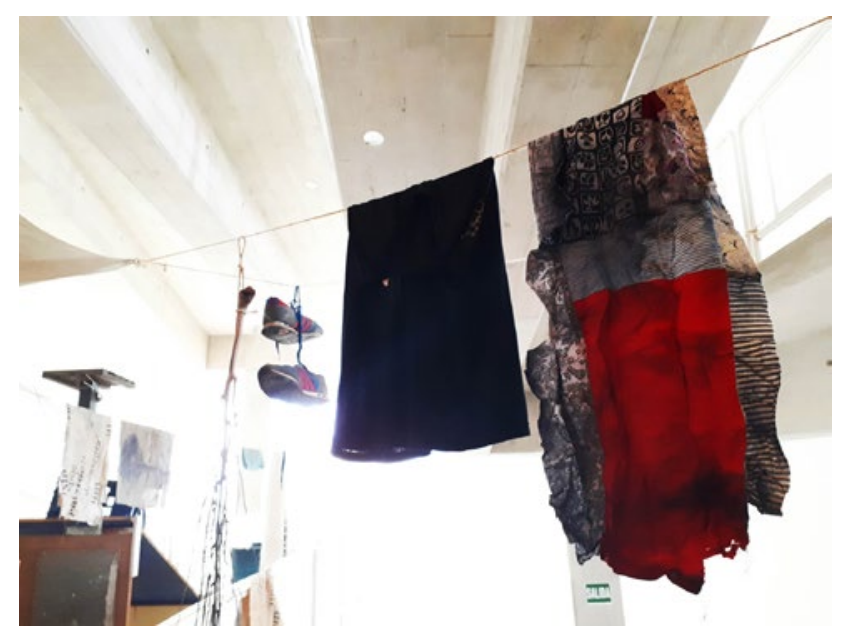

3. Registro de la obra "Lo Que Será Será Y Así Fue". Se observan telas guindadas en cuerdas, en referencia a la ropa que se seca en un patio bajo el sol. Fotografía de Ariane Holz (2017).

\section{ALGUNOS ASPECTOS TEÓRICOS}

Aquello que vive, el hombre o cualquier criatura viva, está en la necesidad, o quizás en la condena, de ocupar un espacio, un territorio para vivir, para habitar.

Las primeras preocupaciones simbólicas de las sociedades políticamente organizadas son el control territorial, la demarcación de sus delimitaciones, y luego, en un segundo lugar, aquello que Foucault (1979) define como biopolítica, el control de la población, de la movilidad y sus flujos. El territorio, entonces, se considera como un resultado de estas relaciones de poder, no solamente dentro del orden de lo espacial o de la cosa, sino, un conjunto de relaciones simbólicas, de actos, de ritmos, sobre el cual se ejerce un control.

El territorio es un espacio que no se usa, sino que se produce. No es un dato cerrado y estático, sino una construcción cultural que se reconfigura. Es un espacio que se apropia y que es dominado por grupos sociales que le otorgan un valor sígnico, a través de los discursos históricos y las memorias colectivas, en función de la construcción de una identidad propia, una identidad que se crea desde la territorialización de los espacios y las demarcaciones del Estado, desde los conceptos de patria y frontera, desde una diferenciación espacial, pero compartiendo prácticas y creencias con otros grupos dentro de las dialécticas culturales.

Estas determinantes identitarias, éstas apropiaciones del territorio, articulan lo que es el habitarreinventar los espacios con una carga simbólica-, o mejor dicho: habitar se convierte en significar. Estos fragmentos espaciales se convierten en significaciones de las comunidades por medio de las relaciones de interdependencias, pues dentro de sus estructuras, no solamente están incluidos los componentes humanos, sino que hay una actividad económica relacionada con campos simbólicos, políticos e ideológicos, que establecen el origen y el arraigamiento entre el individuo y el espacio.

Aquello que constituye al ser humano como persona, en primer lugar, es su identidad, y esa identidad va definida, casi en primera instancia, por una pertenencia territorial. Primero estoy y en tanto soy. Y ahí, claramente, se encuentran esas relaciones de poder, ese control que ejerce el Estado sobre los individuos, unos individuos que están obligados, desde el nacimiento, a pertenecer a una sociedad para significar, a ocupar un espacio -tanto actual como virtual, digamos, por ejemplo, el registro del Estado-, a ser parte de una realidad urbana.

La realidad urbana, así como la realidad rural, o mejor englobado como la realidad del habitar, se constituye a través de las acciones cotidianas y del compromiso político, intelectual, económico y cultural. Esa realidad la hacemos, pero al mismo tiempo, ella nos hace a nosotros. Las aglomeraciones en las ciudades han sufrido cambios extremos desde mediados del siglo XX, en principio, a raíz del aumento de la población en contraste con las áreas rurales. Esto ha sido consecuencia de distintos factores, como la transformación de las ciudades a partir de su industrialización, los éxodos rurales, el aumento de las generaciones en cuestiones de tasas de natalidad y mortalidad, lo que deviene enla expansión de la población, las guerras civiles, la búsqueda de una mejor calidad de vida por los pobladores de zonas rurales, la globalización, la centralización y el derecho a la ciudad.

Si bien, la ciudad es un espacio para la circulación, para la movilidad, esta característica deviene en el éxodo rural, una de las razones primordiales de lo que puede denominarse crisis urbana. En la migración de la población rural a las ciudades, los individuos tienen la oportunidad de ejercer su 
derecho a la ciudad y a la propiedad, el derecho a la vida urbana, al acceso al agua y a los servicios públicos, a permanecer, a no vivir alejados en espacios invisibles ${ }^{2}$ (Correa Montoya, 2010). Los individuos que emigran de las zonas rurales, usualmente explotados y mal pagados, encuentran en las áreas urbanas múltiples posibilidades de elección y la oportunidad de reconocerse como categoría en un lugar donde se hace más posible ejercer sus derechos. Las ciudades latinoamericanas no solamente fueron atractivas para el crecimiento industrial, sino que también fueron receptáculo de las movilidades que acrecentaron el número de la topografía social entre una zona y otra en más del doble, en tan solo una década.

Mike Davis (2004) señala que estas movilidades de grupos sociales a las ciudades fueron el efecto de un ambiente político en América Latina. Por un lado, los planes de ajustes estructurales aplicados por las dictaduras militares desestabilizaron la economía, el empleo y las viviendas, especialmente en las zonas rurales. Por otro lado, los movimientos políticos incitaron a la insurrección rural, y generaron guerras civiles en estas zonas y segregaciones, que se verán igual presentes en las ciudades. En el caso del contexto venezolano, y más específico del estado de Zulia, una causa importante que cabe resaltar fue la explotación del petróleo, que generó un abandono de las periferias para desplazarse a las ciudades con la promesa del progreso.

A través de la selección de una tipología arquitectónica específica, proveniente de las grandes ciudades de los países con dependencia económica y tecnológica de las potencias, la obra (Ver figura 4) propone una reflexión de lo que puede ser la identidad de estas ciudades, pero también, se visibiliza una realidad que es consecuencia de la economía y la industrialización mundial, factores que han generado y masificado las áreas urbanas hiperdegradadas y los asentamientos informales.

Estas áreas urbanas hiperdegradadas se sitúan comúnmente al borde de las explosiones espaciales urbanas, por ejemplo, en ciudades como Caracas, Río de Janeiro o Buenos Aires, por nombrar solo

2 Entenderemos por espacio invisible a los generados por la segregación social, por las políticas excluyentes de las clases sociales marginadas o por la falta de administración eficiente en las periferias urbanas. tres. Mike Davis (2004) expone que la expansión descontrolada de estas áreas constituye un problema en los países con dependencia en la misma medida en que la extensión descontrolada de las áreas residenciales lo es en las potencias.

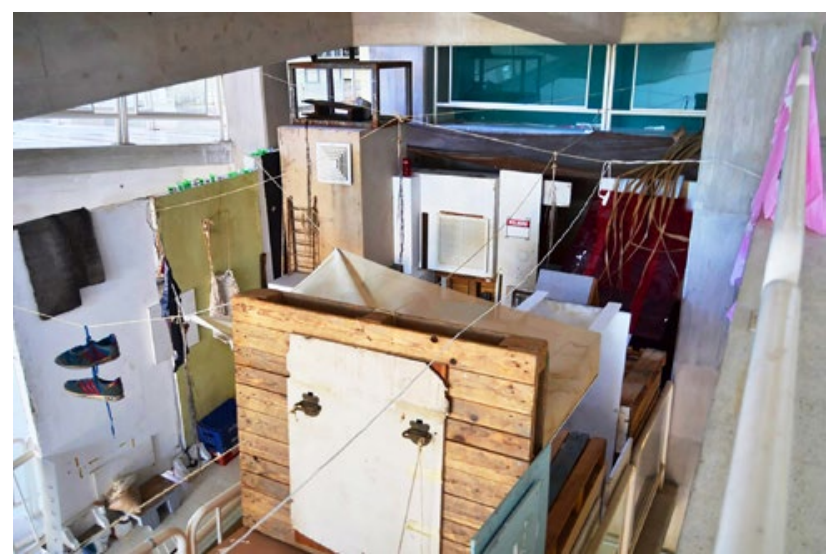

4. Registro de la obra "Lo Que Será Será Y Así Fue" donde se visualiza la reutilización de materiales de desecho y la acumulación de objetos de diferente naturaleza, fotografía de Ángel D. Leiva Valera (2017)

Para muchos nuevos migrantes de zonas pobres que se desplazan a las ciudades, estas zonas precarias son vistas como una oportunidad de establecerse dentro del territorio. En estos asentamientos abundan la laxa propiedad pública, la indeterminación de los títulos de tierra y la especulación.

Los aparatos políticos, según Mike Davis (2004), suelen consentir el asentamiento informal y la especulación privada ilegal, mientras puedan controlar el aspecto político de los asentamientos irregulares y extraigan un flujo regular de sobornos o alquileres. Los individuos que aquí habitan, por no poseer un título de tierra o de propiedad, suelen verse metidos en relaciones de dependencia.

En principio, como dice Foucault (2006), la ciudad no es concebida como una percepción estática, sino que su ordenamiento no puede ser controlado. La megaexpansión y la falta de planificación se hacen presentes en el mismo orden, son consecuencia de la problemática de las políticas urbanas de América Latina dan cabida a que la situación urbana de las zonas hiperdegradadas se siga reproduciendo, y generan microterritorios y movilizaciones dentro de sus callejones, terrazas y viviendas yuxtapuestas unas sobre otras. 


\section{DE LA PERCEPCIÓN DEL TRANSITAR: ASPECTOS SÍGNICOS}

A través de la obra, se busca criticar el aparato estético del museo, el discurso de lo bello y de la representación abstracta, extrayendo un fragmento de la realidad urbana y usándolo como propuesta para crear un discurso sobre la segregación de los grupos sociales y la tipología arquitectónica específica de las clases sociales bajas en los países con dependencia económica y tecnológica de las potencias. Se define un tipo de vivienda, un tipo de espacio, de territorio.

La obra “Lo Que Será Será y Así Fue”, más allá de ser una instalación in situ, busca ser una ambientación ubicada en un espacio museístico. Y, de ser así, no se inscribe la pobreza como elemento semántico dentro de ese ambiente, sino el concepto de insurrección (Ver figura 5).

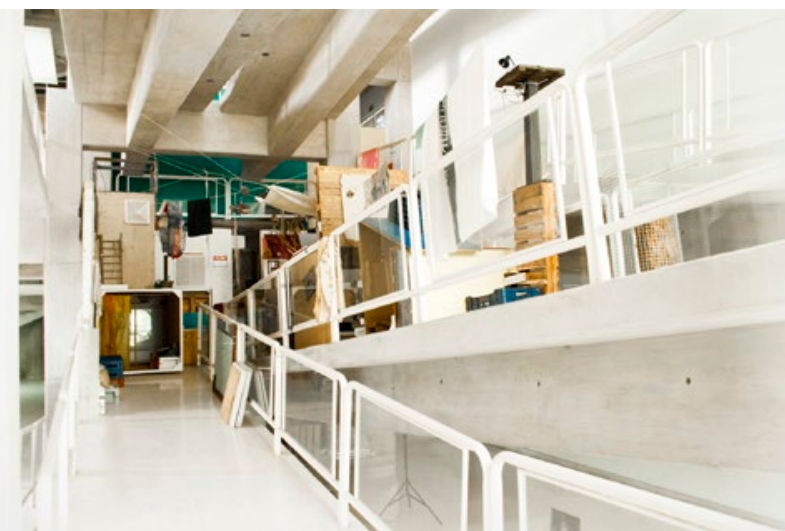

5. Registro de la obra "Lo Que Será Será Y Así Fue" puesta en sala en el Museo de Arte Contemporáneo del Zulia, fotografía de Juan Diego Camacho (2017)

La materialidad de la obra se ubica dentro de un espacio de poder, dentro de una institucionalidad, pero este microterritorio dentro de un territorio mayor -a saber, dentro de un espacio museístico-, busca escapar de la lógica institucional normativizada y deviene en un no-lugar, en una parodia de la realidad de la sala expositiva, y visibiliza el espacio del habitar de un grupo social específico, es decir, las personas en los asentamientos informales, para que los sujetos que transitan en la obra puedan cuestionarse lo que ocurre afuera, puedan evocar fácilmente lo que sus ojos le permiten percibir. Para su construcción, se utilizaron objetos dejados por el museo, lo cual refiere a la manera en que estas casas son construidas, con materiales que sus habitantes encuentran (Ver figura 6).

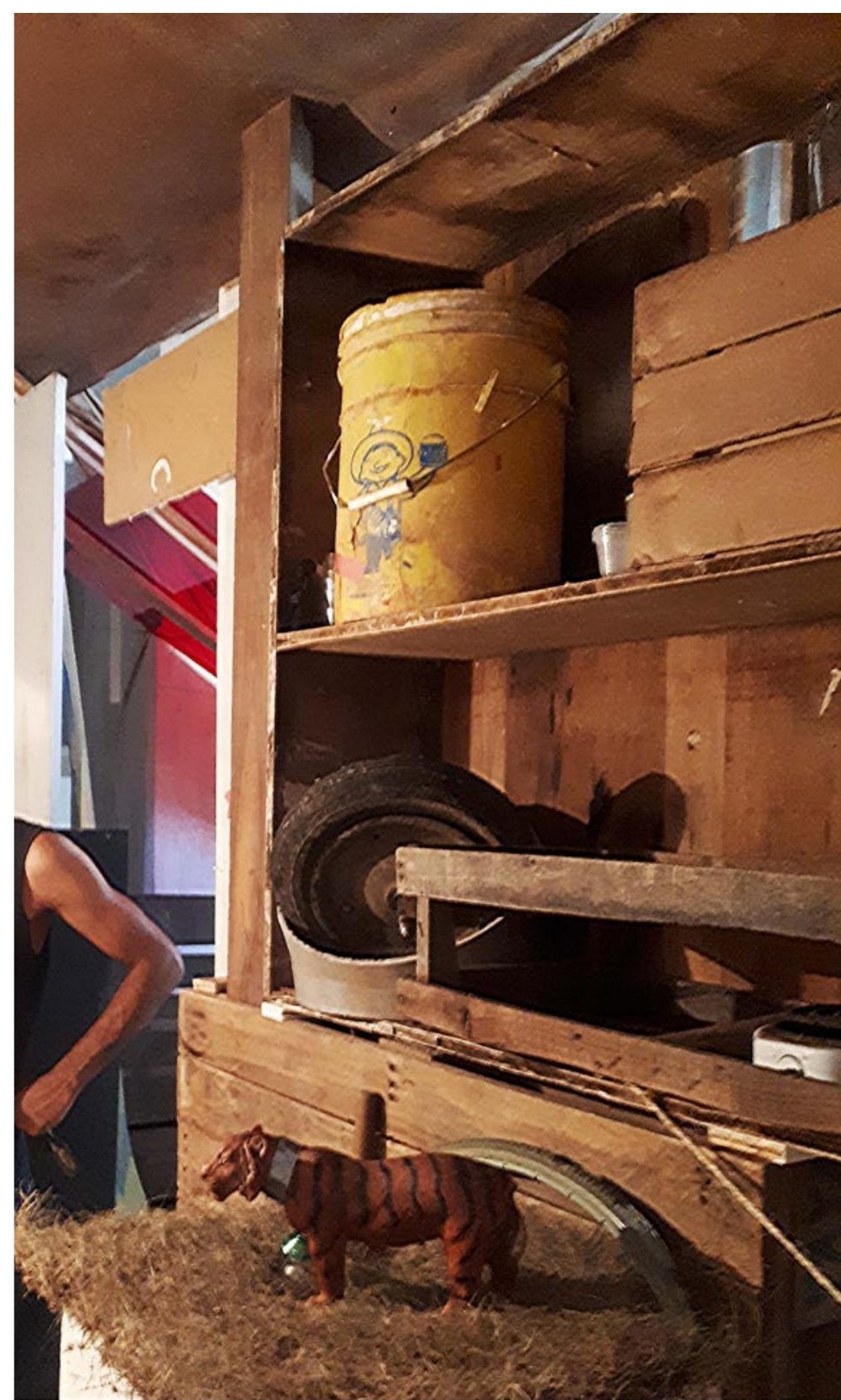

6. Área interior de la obra "Lo Que Será Será Y Así Fue" donde se pueden observar objetos y materiales desgastados que fueron tomados del depósito del museo para la construcción de la estructura, fotografía de Ariane Holz (2017)

Los "no lugares", para Foucault (Spíndola Zago, 2016), son espacios de dialéctica por excelencia, que están permanentemente en conflicto entre las relaciones de poder y la resistencia social, y ponen a prueba la capacidad de adaptación y asimilación de los sujetos. La obra es un recorte geográfico de tiempo, es una fragmentación de la realidad urbana, demuestra sus sistemas, evoca su estructura, permite percibir de cerca una interpretación de esta urbanidad.

El cronotopo, desde la propuesta de Bajtín, es el espacio donde interactúan los elementos de la dimensión espacial con el dominio de lo temporal, e 
y posibilidad de los derechos humanos. Territorios, 22, p. 134-136.

Davis, M. (2004). Planeta de ciudades miseria. Involución urbana y proletariado informal. New Left Review, 26, pp. 15 -16, 21.

Spíndola Zago, Octavio. (2016). Espacio, territorio y territorialidad: una aproximación teórica a la frontera. Revista mexicana de ciencias politicas y sociales, 61(228), p. 27-56. Recuperado en 10 de abril de 2018, de http://www. scielo.org. $\mathrm{mx} /$ scielo.php?script=sci arttext\&pid=S0185-19182016000300027\&ln$\mathrm{g}=\mathrm{es} \& \mathrm{t} \ln \mathrm{ln}=\mathrm{es}$. 